\title{
Increasing Resident Support Following Patient Suicide: Assessing Resident Perceptions of a Longitudinal, Multimodal Patient Suicide Curriculum
}

\author{
Samar McCutcheon ${ }^{1}$ (D) Julie Hyman ${ }^{1}$ \\ Received: 26 August 2020 / Accepted: 18 February 2021/Published online: 2 March 2021 \\ (C) Academic Psychiatry 2021
}

\begin{abstract}
Objective Patient suicide is a common experience in psychiatry residency, and its effects on trainees can be profound. There are currently no ACGME Common Program Requirements for education about patient suicide, and a need exists for evidence-based curricula to prepare residents for this difficult outcome.

Methods A comprehensive patient suicide curriculum was developed utilizing multiple modes of delivering content, including a training designed to foster built-in support among peers in the healthcare workplace. The content was delivered at intervals over the course of the 2019-2020 academic year for 43 psychiatry residents at The Ohio State University Wexner Medical Center. Preand post-curriculum surveys were obtained to assess the resident experience of the new curriculum.

Results Twenty-seven residents completed the pre-curriculum survey and 25 completed the post-curriculum survey. Results demonstrated statistically significant improvements in ratings of preparedness to deal with the loss of a patient by suicide, preparedness to support a co-resident who has experienced the death of a patient by suicide, program-level support for residents, understanding systems-level and quality processes, and knowledge of what steps to take if finding out a patient has completed suicide.

Conclusions A multimodal approach incorporating understanding emotional reactions, provision of support, delineation of procedural issues, and education regarding quality and risk management considerations was effective at improving resident preparedness to cope following a patient suicide.
\end{abstract}

Keywords Patient suicide $\cdot$ Patient suicide curriculum $\cdot$ Postvention

The study of psychiatrist reactions to patient suicide is often traced back to a 1965 essay by Litman [1] in which he states: "One might expect that therapists would express more philosophic attitudes when asked to consider the possibility of death as an abstraction. Yet the same persons might describe quite different personal emotional experiences after a direct encounter with the death as an actual event" (p. 570). While the loss of a patient to suicide can be devastating for any psychiatrist, it may be particularly traumatizing for trainees [2]. Patient suicide is cited as one of the most stressful adversities experienced during residency training [3]. Unfortunately, it is not uncommon for residents to experience

Samar McCutcheon

samar.mccutcheon@osumc.edu

1 The Ohio State University College of Medicine, Columbus, $\mathrm{OH}$, USA patient suicide: a systematic review of 8 worldwide surveys found rates of patient suicide experienced by psychiatry residents ranged between 31 and $69 \%$. Of note, 3 of the 8 surveys analyzed were national or multisite and reported rates at the higher end of this range (47-69\%); however, only 1 of the 8 studies meeting the inclusion criteria was based in the USA and reported a $33 \%$ incidence [4].

Despite the high rates of patient suicide, there has been little formal education or intervention (otherwise known as post-invention) provided to residents who experience patient suicide [5]. There is no current requirement for residency training in patient suicide by the Psychiatry Common Program Requirements [6]. As of 1998, less than half of the programs in a national survey instructed residents on what to do if a patient commits suicide [7]. In 2003, Pilkinton et al. found that a third of psychiatry residents surveyed received education on the impact of suicide on trainees and a third of program directors had a policy in place for resident debriefing [8]. By 2009, a survey of chief residents indicated that $25 \%$ of 
programs covered topics related to patient suicide, suggesting that education in patient suicide remains lacking [9]. The importance of formally addressing patient suicide is highlighted in a survey of psychiatrists who experienced patient suicide in training: $71 \%$ felt helpless, $69 \%$ reported the patient suicide had a significant impact on them, $55 \%$ experienced recurrent feelings of horror, and $44 \%$ developed significant anxiety following the event [10].

To better prepare psychiatry residents at The Ohio State University Wexner Medical Center for the possible experience of patient suicide during training, we explored existing research to guide development of a patient suicide curriculum. In a survey with 239 responding graduates in practice and trainees of the residency program at University of Toronto, Ruskin et al. found that $16 \%$ experienced patient suicide by the end of the first year of residency training, suggesting that the curriculum should begin early in the academic year [9]. Brown recommends that a training director or supervisor meet with the resident almost immediately after a patient suicide to provide support [11]. This intervention is supported by reports that residents experienced a personal reduction of guilt when supervisors shared their own responsibility and guilt [5]. An absence of this support has also been shown to contribute to increased trainee distress [12]. In addition to training director and supervisor support, the role of peer support dates back to a letter by Binder in 1978 [13], and 64\% of trainees experiencing patient suicide found the support of their peers to be helpful [10].

There is existing data to support the use of formal training to deliver a patient suicide curriculum. Lerner et al. shared outcomes from a biennial half-day workshop on medical-legal issues and coping skills for patient suicide [14]. Results demonstrated statistically significant increases in ratings of comfort supporting a colleague who experienced patient suicide. Whitmore et al. reported on outcomes from a 4-h course on safety assessment and patient suicide during intern orientation [15]. Results showed a statistically significant improvement in rating of ability to support a peer following patient suicide.

\section{Methods}

After reviewing existing research, a new multimodal curriculum to ensure residents have sufficient education about patient suicide and knowledge of postvention supports was developed and implemented within the adult general psychiatry residency at The Ohio State University Wexner Medical Center. The curriculum began in July 2019, with a mandatory Brief Emotional Support Team (BEST) training experience for all residents and faculty involved in resident supervision. BEST training is a 1.5-h course that uses evidence-based training and therapy customized to assist first responders and healthcare professionals to respond effectively in a crisis while also engaging in skills that build resilience to cope with chronic exposure to stress [16]. This training was initially developed by the Stress Trauma and Resiliency team at The Ohio State University Wexner Medical Center to provide groups of co-workers with a pre-emptive framework to recognize the need for and ways to address trauma among themselves. BEST training equips groups with tools and skills to develop and nurture a culture of built-in support among peers in the workplace. It was selected to strengthen the peer and supervisor support that could be provided to residents who experienced patient suicide.

In August, a communication tree was disseminated to all faculty, residents, and staff (available upon request). This tree clearly outlines the necessary steps to follow if anyone (e.g., faculty, resident, staff, nursing) receives notification that a patient has completed suicide. It formalizes the procedure for delivering the news to a resident in an effort to ensure a supervisor and/or training director is able to provide immediate support. In September, a 1-h faculty panel comprised of supervisors who had experienced a patient suicide during training was convened for all residents. This allowed residents an opportunity to hear faculty share their personal experiences with patient loss and engage in an open dialogue if trainees had questions about the experience. In October and November, a 2-part article by Nina Gutin, $\mathrm{PhD}$ was disseminated by email to the residents. The first article, "Losing a patient to suicide: What we know," reviewed "the impact of losing a patient to suicide, confidentiality-related constraints on the ability to discuss and process the loss, legal and ethical issues, colleagues' reactions and stigma and the effects of a suicide loss on one's clinical work" [17]. The second article, "Losing a patient to suicide: Navigating the aftermath," discussed "the opportunities for personal growth that can result from experiencing a suicide loss, guidelines for optimal postventions, and steps clinicians can take to help support colleagues who have lost a patient to suicide" [18]. The final portion of the curriculum took place in February when all residents met with an attorney from the hospital's Risk Management division and a faculty member who serves as the department's Associate Chief Quality Officer. This interactive discussion session reviewed the root cause analysis process conducted after a patient suicide, as well as the medical-legal implications.

This curriculum was conducted in a large, academic psychiatry department. Eighteen faculty members participated in the BEST training, and 43 residents (14 PGY-1, 11 PGY-2, 11 PGY-3, and 7 PGY-4) participated in all components of the curriculum. The Institutional Review Board reviewed the study and gave it exempt status.

To assess the effectiveness of the curriculum, a 10-question anonymous electronic survey was generated via MedHub. This survey was distributed on July 10, 2019, and again on June 1, 2020, to all faculty and residents. While the curriculum was completed in February, an end-of-year distribution for the 
final survey was selected to best capture the number of residents experiencing patient suicide over the course of the academic year. With each survey distribution, 3 reminder emails were sent to non-responders. The first 2 survey questions asked for respondent year of training and if the respondent had experienced the death of a patient by suicide during training. Respondents who had experienced the death of a patient by suicide were asked who they relied on for support. All respondents were asked if they were aware of the resources available for support after the death of a patient by suicide. The remaining six questions utilized a Likert scale of 1 to 5 (1 = strongly disagree; 2 = disagree; $3=$ neutral; 4 = agree; 5 = strongly agree) and are listed in Table 1. For survey analysis, we calculated the mean and standard deviation of scores on the Likert scales. We used an unpaired $t$ test to compare preand post-curriculum values with GraphPad Instat 3 (GraphPad Software, San Diego, CA).

\section{Results}

The pre-curriculum survey was completed by 27 residents (63\% response rate), and 25 residents (58\% response rate) completed the post-curriculum survey. At the initial survey, 3 of 27 residents (11\%) reported having experienced the death of a patient by suicide in their training. Respondents reported reaching out to co-residents, family, attendings, and graduates of the program for support. Of respondents, 17 of 27 (63\%) residents were aware of the resources available for support after the death of patient by suicide. On the end-of-year survey, 4 of 25 residents (16\%) reported having experienced the death of a patient by suicide. Similarly, respondents reported reaching out to co-residents, family, attendings, and the program director. The number of residents aware of available resources increased to 23 of 25 residents (92\%).

Table 1 summarizes the mean with standard deviation for the survey items rated by Likert scale and corresponding $P$ values. On analysis, several survey items demonstrated statistically significant changes from pre- to post-curriculum. These included ratings of preparedness to deal with the loss of a patient by suicide, preparedness to support a co-resident who has experienced the death of a patient by suicide, programlevel support for residents following a patient suicide, understanding of the systems-level and quality processes that occur after a patient suicide, and knowledge of what steps to take if finding out a patient has completed suicide. The change in perception that losing a patient to suicide would negatively impact patient care was not statistically significant.

\section{Discussion}

Death of a patient by suicide is an unfortunately common experience during psychiatry residency training [4]. As the rate of suicide in the USA has increased to $24 \%$ from 1999 to 2014 , it is more critical than ever for psychiatry residency programs to prepare trainees for this possible outcome [19]. Currently, few psychiatry residency programs offer formal education on patient suicide and a need exists for an evidence-based curriculum to address this difficult experience [8].

Review of the available literature on the limited existing patient suicide curricula shows a focus on common topics, including emotional reactions to patient suicide, available support resources, procedures to follow after a patient suicide, interactions with the family of the deceased patient, root cause analysis process, and malpractice concerns [14, 15, 20]. Likewise, our curriculum addressed these areas, as well. BEST training covered common emotional reactions, how to support peers, and available resources for additional support. The communication tree outlined procedures to follow upon learning the news of a patient suicide. The quality and risk management session specifically addressed the sentinel event process and liability concerns. The faculty panel and disseminated articles further reinforced the information.

Although other programs have consolidated the information into single workshops $[14,15,20]$, the various

Table 1 Postvention curriculum surveys

\begin{tabular}{|c|c|c|c|}
\hline Survey items & $\begin{array}{l}\text { Pre-curriculum survey } \\
\text { Mean } \pm \text { SD (scale 1-5) }\end{array}$ & $\begin{array}{l}\text { Post-curriculum survey } \\
\text { Mean } \pm \text { SD (scale 1-5) }\end{array}$ & $P$ value \\
\hline $\begin{array}{l}\text { My training program has adequately prepared me to deal } \\
\text { with the loss of a patient to suicide. }\end{array}$ & $3.33 \pm 0.9$ & $4.08 \pm 0.8$ & $<.001$ \\
\hline $\begin{array}{l}\text { I feel prepared to support a co-resident who has experienced } \\
\text { the death of a patient by suicide }\end{array}$ & $3.59 \pm 0.8$ & $4.32 \pm 0.7$ & .001 \\
\hline The residency program is supportive following a patient suicide. & $3.93 \pm 0.7$ & $4.40 \pm 0.6$ & .01 \\
\hline $\begin{array}{l}\text { I understand the systems-level and quality processes that occur } \\
\text { after a patient suicide. }\end{array}$ & $3.41 \pm 1.1$ & $4.08 \pm 0.9$ & .02 \\
\hline $\begin{array}{l}\text { I know what steps to take if I find out one of my patients has } \\
\text { completed suicide, including who to contact. }\end{array}$ & $3.19 \pm 1.0$ & $4.08 \pm 1.0$ & .002 \\
\hline $\begin{array}{l}\text { Losing a patient to suicide would negatively impact my ability } \\
\text { to continue providing care to my patients. }\end{array}$ & $2.96 \pm 1.0$ & $2.88 \pm 1.0$ & .68 \\
\hline
\end{tabular}


components of this curriculum were scheduled at intervals throughout the year. The goal of this scheduling was to allow time for residents to process the information and to keep the topic at the forefront of training throughout the year. This curriculum is also unique in its use of multiple modes of presenting the material (lecture, panel discussion, dissemination of written material, interactive discussion session) in order to meet the needs of different learning styles.

The pre-curriculum survey results suggested opportunity for improving resident preparedness in multiple domains related to patient suicide. The post-curriculum survey results indicate the patient suicide curriculum implemented with psychiatry residents at The Ohio State University Wexner Medical Center was successful at improving resident ratings of preparedness to deal with patient suicide and ability to support a peer experiencing patient suicide. The curriculum also increased perceptions of program-level support for residents and understanding of the events occurring after patient suicide (systems-level, quality processes, and knowledge of what steps to take if finding out a patient has completed suicide). The perception that losing a patient to suicide would negatively affect patient care was the only survey item that did not demonstrate statistically significant improvement. This suggests that a trainee's confidence in their ability to provide patient care after a bad outcome may need to be addressed more explicitly in the curriculum.

A strength of this study was the number of survey responses available for review. Although a sizable number of residents responded to the surveys, the overall response rate itself could have been higher, which is a limitation to interpreting this data. It is possible that residents who did not find the curriculum helpful chose not to respond to the survey, and it is possible residents experienced survey fatigue with increased distribution of other surveys related to COVID-19. Other limitations include implementing the curriculum at a single academic site, the lack of a control group, and the use of subjective preparedness ratings rather than ratings in the face of a completed patient suicide. Further areas for research include examining which components of the curriculum were most helpful for the individual items on the survey and whether the curriculum continues to benefit residents after training as they encounter patient suicide in their professional careers.

Acknowledgments We thank the residents, Dr. Julie Niedermier, Dr. Anne-Marie Duchemin, and Dr. Kenneth Yeager for their contribution to this project

\section{Declarations}

Ethical Approval The research was approved by the Biomedical Institutional Review Board.
Disclosures On behalf of all authors, the corresponding author states that there is no conflict of interest.

\section{References}

1. Litman RE. When patients commit suicide. Am J Psychother. 1965;19:570-6.

2. Chemtob CM, Hamada RS, Bauer G, Kinney B, Torigoe RY. Patients' suicides: frequencies, and impact on psychiatrists. Am J Psychiatry. 1988;145:224-8.

3. Kozlowska K, Nunnn K, Cousens P. Adverse experiences in psychiatry training, part 2. Aust N Z J Psychiatry. 1997;31:641-52.

4. Puttagunta R, Lomax ME, McGuiness JE. What is the prevalence of the experience of death of a patient by suicide among medical students and residents? A systemic review. Acad Psychiatry. 2014;38:538-41.

5. Schnur DB, Levin EH. The impact of successfully completed suicides on psychiatric residents. J Psychiatr Educ. 1985;9:127-36.

6. Psychiatry. ACGME Main Page. https://www.acgme.org/ Specialties/Program-Requirements-and-FAQs-and-Applications/ pfcatid/21/Psychiatry. Published July 1, 2020. Accessed February 3, 2021.

7. Ellis TE, Dickey TO, Jones ED. Patient suicide in psychiatry resident programs: a national survey of training and postvention practices. Acad Psychiatry. 1998;22:181-9.

8. Pilkinton P, Etkin M. Encountering suicide: the experience of psychiatric residents. Acad Psychiatry. 2003;27:93-9.

9. Melton B, Coverdale J. What do we teach psychiatric residents about suicide? A national survey of chief residents. Acad Psychiatry. 2009;33:47-50.

10. Ruskin R, Sakinofsky I, Bagby RM, Dickens S, Sousa G. Impact of patient suicide on psychiatrists and psychiatric trainees. Acad Psychiatry. 2004;28:104-10.

11. Brown HN. Patient suicide during residency training (I): incidence, implications, and program response. Acad Psychiatry. 1987;11: 201-16.

12. Lafayette JM, Stern TA. The impact of a patient s suicide on psychiatric trainees: a case study and review of the literature. Harv Rev Psychiatry. 2004;12:49-55.

13. Binder R. Dealing with patients' suicides [Letter]. Am J Psychiatry. 1978; 135:1113.

14. Lerner U, Brooks K, Mcniel DE, Cramer RJ, Haller E. Coping with a patient's suicide: a curriculum for psychiatry residency training programs. Acad Psychiatry. 2012;36:29-33.

15. Whitmore CA, Cook J, Salg L. Supporting residents in the wake of patient suicide. Am J Psychiatry Resid J. 2017;12:5-7.

16. For Professionals - STAR I Ohio State College of Medicine. College of Medicine. https://medicine.osu.edu/departments/ psychiatry-and-behavioral-health/star/for-professionals. Accessed February 3, 2021.

17. Gutin NJ. Losing a patient to suicide: what we know. Curr Psychiatry. 2019;18:15-32.

18. Gutin NJ. Losing a patient to suicide: navigating the aftermath. Curr Psychiatry. 2019;18:17-24.

19. Curtin SC, Warner M, Hedegaard H. Increase in suicide in the United States, 1999-2014. NCHS Data Brief. 2016;241:1-8.

20. Prabhakar D, Balon R, Anzia JM, Gabbard GO, Lomax JW, Bandstra BSY, et al. Helping psychiatry residents cope with patient suicide. Acad Psychiatry. 2014;38:593-7.

Publisher's Note Springer Nature remains neutral with regard to jurisdictional claims in published maps and institutional affiliations. 